\title{
An Ultra-Fast Digitally Reconstructed Radiograph (DRR) Implementation of the Siddon-Jacobs Algorithm using Parallel Computing: Runtime Improvement of an Intensity-Based 2D/3D Registration
}

\begin{abstract}
A key component of an intensity-based 2D/3D registration is the digitally reconstructed radiograph (DRR) module, which creates $2 \mathrm{D}$ projections from pre-operative $3 \mathrm{D}$ data, e.g., CT and MRI scans. On average, an intensity-based $2 \mathrm{D} / 3 \mathrm{D}$ registration requires ten iterations and the rendering of twelve DRR images per iteration. In a typical DRR implementation, the rendering time is about two seconds, and the registration runtime is four minutes.

We present an implementation of the Siddon-Jacobs algorithm that uses a novel pixel-step approach to determine the pixel location of the rendering plane. In addition, we calculate the intensity of each pixel in the rendering plane using a parallel computing approach. The DRR rendering time is reduced to $10 \mathrm{~ms}$ on average so that the registration runtime can be achieved in an average of 4.8 seconds.
\end{abstract}

Keywords: DRR, intensity-based, 2D/3D, registration, parallel computing, imaging modalities.

https://doi.org/10.1515/cdbme-2021-2007

\section{Introduction}

Intensity-based $2 \mathrm{D} / 3 \mathrm{D}$ registration is an iterative method of bringing pre-operative 3D data, e.g., CT-Scans or MRIs in DICOM format, into the operating room using conventional

\footnotetext{
*Corresponding author: Julio C. Alvarez-Gomez: Institute of Control Engineering and Center for Sensor Systems (ZESS), University of Siegen, Siegen, Germany, e-mail: julio.agomez@uni-siegen.de.

Gerardo Jimenez Palavicini, Hubert Roth and Jürgen

Wahrburg: Institute of Control Engineering and Center for Sensor Systems (ZESS), University of Siegen, Siegen, Germany
}

intra-operative modalities such as radiographs from a mobile $\mathrm{X}$-ray device (C-arm). A key component of an intensity-based registration is the digitally reconstructed radiograph (DRR) module, which creates $2 \mathrm{D}$ projections from the pre-operative 3D data. Our implementation of the intensity-based 2D/3D registration requires the rendering of twelve DRR images per iteration and an average of ten iterations to complete the process. In typical DRR implementations in medical frameworks, e.g., ITK and MeVisLab, the rendering time is about two seconds. The registration runtime is four minutes using DRR rendering sizes of $142 \times 142$ pixels. Some fast DRR approaches based on CPU multi-threading [1] and non-ray casting methods, namely attenuation fields [2], have been previously reported.

In this paper, we present an ultra-fast implementation of the Siddon-Jacobs algorithm. The improvements are achieved by I) accelerating the calculation of the pixels in the rendering plane with a novel pixel-step approach II) computing the intensity of each pixel in the rendering plane using a parallel computing approach on the GPU. The tests are performed using the CT-Scan of a spine phantom with a size of $512 \times 512 \times 442$ voxels and the DRR rendering size of $568 \times 568$ pixels, the same image dimension of an X-ray image of the Carm available in our laboratory.

\section{Materials and Methods}

A C-arm consists of an X-ray source at one end of its C-shape structure and an X-ray detector at the other end. The radiation pierces a patient lying in the $\mathrm{C}$-arm, and the detector forms an $\mathrm{X}$-ray image by capturing the attenuated radiation due to the body segment. Now imagining a C-arm in the digital world, but instead of a patient, there is a DICOM volume, e.g., a CTscan imaging. The rays go through the DICOM and generate 
an X-ray image out of this $3 \mathrm{D}$ volume. Thus, the resulted $\mathrm{X}$ ray image is instead called a digitally reconstructed radiograph (DRR). In the DRR module, the ray source is punctual and is located in a known position with respect to the $3 \mathrm{D}$ object. Then the resulted image is created with a perspective projection. The nature of the DRR is equivalent to that of the $\mathrm{C}$-arm. It can be noticed that the reference frame of the DRR module and the DICOM belongs to a three-dimensional space, so the description of the object with respect to the DRR frame requires six degrees of freedom (DoF): three positions and three orientations. This six DoF are gathered in the vector $\overrightarrow{\boldsymbol{p}} \in \mathbb{R}^{6}$ described in in equation (1).

$$
\vec{p}=\left[t_{x}, t_{y}, t_{z}, r_{x}, r_{y}, r_{z}\right]^{T}
$$

For radiological applications, the first ray casting algorithm was the Siddon algorithm [3]. The algorithm performs the volume ray casting by establishing the equation for the radiological path as:

$$
d=\sum_{i} \sum_{j} \sum_{k} l(i, j, k) \rho(i, j, k)
$$

where $\rho(i, j, k)$ is the voxel intensity value and $l(i, j, k)$ the ray length contained by that voxel.

A faster version of the Siddon algorithm was achieved from Jacobs in 1998, creating the known Siddon-Jacobs algorithm. The implementation is reported to speed up the computation time by 7.5 in comparison with the Siddon algorithm [4]. The Siddon-Jacobs algorithm calculates the intersects of the radiological path and the CT-Scan volume using a set of geometrical relations using the ray entry and exit points in the volume. The Siddon-Jacobs algorithm can be found in some medical frameworks like ITK and MeVisLab. We use the MeVisLab framework as the base for the $2 \mathrm{D} / 3 \mathrm{D}$ registration approach. Consequently, we used the DRR native module in $\mathrm{MeVisLab}$ as the ground-truth for the image rendering and runtime measure.

Our implementation uses the Siddon-Jacobs algorithm to render a DRR image. For changing the DICOM pose with respect to the DRR reference frame (1), we implemented an approach called pixel-step method. In the DRR module, the origin is located equidistance along the $\mathrm{Y}$-axis between the source and the detector. The ray-source is represented by point $\boldsymbol{s}=[0, f / 2,0]$, and the detector is represented by three points $\boldsymbol{a}, \boldsymbol{b}$, and $\boldsymbol{c}$, which defined three corners of the detector. As $\boldsymbol{s}, \boldsymbol{a}, \boldsymbol{b}$, and $\boldsymbol{c}$ are described by the DRR frame, they can be written as ${ }^{D R R} \boldsymbol{p}_{s},{ }^{D R R} \boldsymbol{p}_{a},{ }^{D R R} \boldsymbol{p}_{b}$, and ${ }^{D R R} \boldsymbol{p}_{c}$, representing the corners of the resulted image in $\mathbb{R}^{3}$. The points ${ }^{D R R} \boldsymbol{p}_{s},{ }^{D R R} \boldsymbol{p}_{a},{ }^{D R R} \boldsymbol{p}_{b}$, and ${ }^{D R R} \boldsymbol{p}_{c}$ are the elements, where the transformation ${ }^{D R R} \boldsymbol{T}_{\text {DICOM }}$ is applied. Once the new position of those points is achieved, i.e., ${ }^{\text {DICOM }} \boldsymbol{p}_{s},{ }^{\text {DICOM }} \boldsymbol{p}_{a},{ }^{\text {DICOM }} \boldsymbol{p}_{b}$, and ${ }^{\text {DICOM }} \boldsymbol{p}_{c}$, each pixel of the image can be found by adding offsets of pixel spacing sizes in X-and Z-axis in $\{D I C O M\}$. The pixel $(n, m)$ of the DRR image can be calculated using the equation (1) and (2) with the initial point, ${ }^{D I C O M} \boldsymbol{p}_{s}$, and the final point ${ }^{D I C O M} \boldsymbol{p}_{\text {PIXEL }_{-}(n, m)}$. The above procedure is illustrated in Figure 1.

The Siddon-Jacobs algorithm was created initially for serial computing, but its working principle allows parallelization, which leads to an increment in the computing speed. For every pixel, the initial and final points require for the ray-casting algorithm are calculated using the pixel-step method, and then the Siddon-Jacobs algorithm is applied by calculating the line integral. Using a parallel approach, several pixels can be calculated simultaneously using the computational power of the graphics processing unit (GPU) as the calculation of one pixel is independent of any other pixel. Intrinsically, the GPU is designed for high-speed graphics,
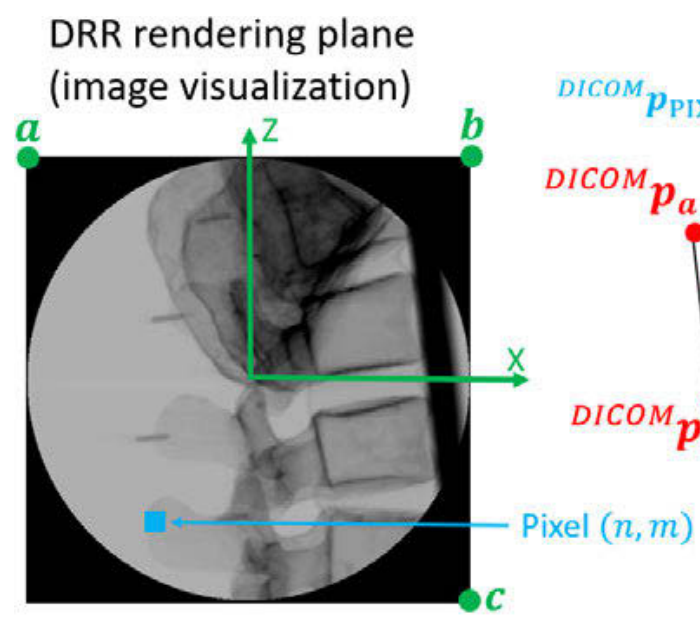

\section{DRR rendering plane} (1) 
An Ultra-Fast Digitally Reconstructed Radiograph (DRR) Implementation of the Siddon-Jacobs Algorithm using Parallel Computing:

which are inherently parallel [5]. While CPUs focus on low latency, GPUs focus on high throughput. That means the CPU is faster than the GPU to accomplish a task, but the GPU is faster to process thousands of tasks due to its parallel capabilities. With the Compute Unified Device Architecture (CUDA) release by NVIDIA in 2006, GPUs were brought into the general-purpose processing applications [6].

CUDA is a parallel computing platform that allows defining $\mathrm{C}++$ functions, called kernels, which are executed $N$ times in parallel by $N$ different CUDA threads [6]. In our research, an Nvidia GTX 1080 graphics card is used, which can execute 16 threads per CUDA core, and it has 2560 CUDA cores. Consequently, the GPU can execute up to 40960 threads simultaneously. Since the GPU processor frequency, $1.57 \mathrm{Ghz}$ is 2.4 times slower than the used CPU, 3.7Ghz, the computation of a single-pixel of the DRR image takes longer using a GPU thread. However, the vast number of simultaneous pixels the GPU can process is enough to increase the overall execution time.

\section{Results}

The vector $\overrightarrow{\boldsymbol{p}}$ expresses the transformation from $\{\mathrm{DRR}\}$ to $\{\mathrm{DICOM}\}$, also represented as ${ }^{D R R} \boldsymbol{T}_{\text {DICOM }}$. The ray-casting algorithm goes through the volume and computes the line integral using the voxels in its path, eq. (2). Consequently, the computation time depends on the number of voxels to evaluate, which are a function of the CT-scan size and the ray trajectory that depends on $\overrightarrow{\boldsymbol{p}}$. Figure 2 depicts the difference in the ray-path length inside the volume depending on $\overrightarrow{\boldsymbol{p}}$. $\overrightarrow{\boldsymbol{p}}_{\mathbf{1}}$ depicts a shorter path than $\overrightarrow{\boldsymbol{p}}_{2}$, which translates into a shorter rendering time for $\overrightarrow{\boldsymbol{p}_{\mathbf{1}}}$ than $\overrightarrow{\boldsymbol{p}}_{\mathbf{2}}$.

Our registration procedure uses X-ray images of $568 \mathrm{x} 568 \mathrm{px}$. As the DRR images are compared with the X-ray images, they are generated with the same resolution. The results of the performance test using the ground-truth DRR module and a CT-Scan of a spine phantom of $512 \times 512 \times 442$ voxels can be seen in Table 1 .
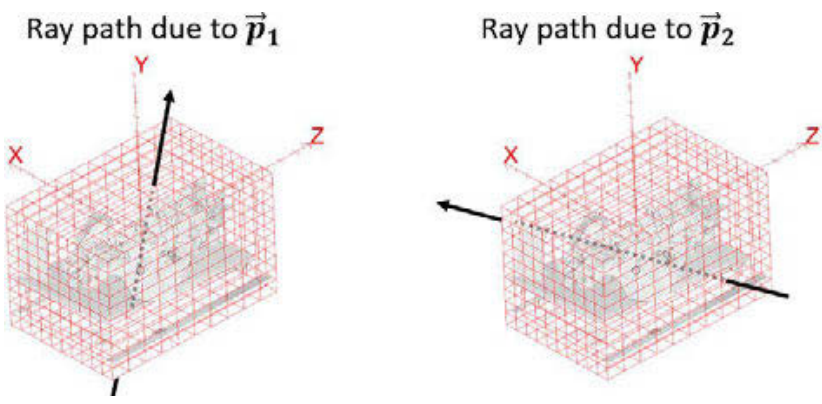

Figure 2: Ray path dependency with $\overrightarrow{\boldsymbol{p}}$
Table 1: Performance of the ground-truth DRR module

\begin{tabular}{ccccc|c|c|cc}
\hline & \multicolumn{7}{c}{$\vec{p}$} & \\
\cline { 2 - 7 } Test & \multicolumn{3}{c}{$[\mathrm{mm}]$} & \multicolumn{5}{c}{ ['] } \\
\cline { 2 - 7 } & $\boldsymbol{t}_{\boldsymbol{x}}$ & $\boldsymbol{t}_{\boldsymbol{y}}$ & $\boldsymbol{t}_{\boldsymbol{z}}$ & $\boldsymbol{r}_{\boldsymbol{x}}$ & $\boldsymbol{r}_{\boldsymbol{y}}$ & $\boldsymbol{r}_{\boldsymbol{z}}$ & \\
\hline 1 & 0 & 0 & 0 & 0 & 0 & 0 & 16.12 \\
2 & 20 & 20 & 20 & 45 & 0 & 0 & 20.14 \\
3 & 50 & 50 & 0 & 0 & 0 & 45 & 21.66 \\
4 & 10 & 20 & 30 & 45 & 0 & 45 & 32.85 \\
\hline
\end{tabular}

A DRR image is rendered after the $N \times M$ pixels of the image are calculated. In this particular case, the created images consist of $568 \times 568$ pixels, which compute 322624-line integrals, one per pixel. In the center of Figure 3, it can be seen a DRR image created with the own implementation; on the left side, it can be seen the DRR image from the ground-truth module using the same $\overrightarrow{\boldsymbol{p}}$. Both images have the grayscale range $\{0-255\}$. The pixel-wise absolute difference between both images is calculated and displayed on the right side of Figure 3. The darker the resulted image, the more similar the two DRR images are. It can be noticed that consistent gray background color and some grayscale patches on the body segment are visible. The patches are due to approximation errors and edge handling between implementations. The whitest spots visible in the pixel-wise absolute difference image have a grayscale value of 11 , while the darkest spots have a grayscale value of 4 . In the visualization of the difference image, the contrast was increased to the maximum; otherwise, it would not have been possible to visualize the difference as the grayscale range is $\{4-11\}$. This reduced grayscale range is a good indicator of the similarity between the two implementations.

Table 2 compares the performance between the groundtruth and the parallel computing implementation of the DRR. Now the average rendering times are around $10 \mathrm{~ms}$, showing an improvement of 2882 times with the ground-truth, more than three orders of magnitude.

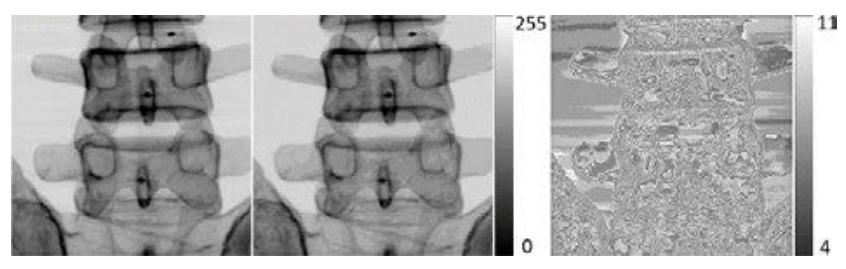

Figure 3: DRR image rendering: Left ground truth, center own implementation. Pixel-wise absolute difference between DRR images on the right 
Table 2: Performance of the parallel implementation (PC) compared with the ground-truth (GT) DRR module

\begin{tabular}{c|c|c|c|c|c|c|c|c}
\hline & \multicolumn{7}{c|}{$\overrightarrow{\boldsymbol{p}}$} & \multicolumn{3}{c}{ Time [ms] } \\
\cline { 2 - 7 } Test & \multicolumn{3}{|c|}{ [mm] } & \multicolumn{3}{c}{ ['] } & PC \\
\cline { 2 - 7 } & $\boldsymbol{t}_{\boldsymbol{x}}$ & $\boldsymbol{t}_{\boldsymbol{y}}$ & $\boldsymbol{t}_{\boldsymbol{z}}$ & $\boldsymbol{r}_{\boldsymbol{x}}$ & $\boldsymbol{r}_{\boldsymbol{y}}$ & $\boldsymbol{r}_{\boldsymbol{z}}$ & & \\
\hline 1 & 0 & 0 & 0 & 0 & 0 & 0 & 16120 & 4.1 \\
2 & 20 & 20 & 20 & 45 & 0 & 0 & 20140 & 5.98 \\
3 & 50 & 50 & 0 & 0 & 0 & 45 & 21660 & 6.57 \\
4 & 10 & 20 & 30 & 45 & 0 & 45 & 32850 & 22.8 \\
\hline
\end{tabular}

These rendering times in the $2 \mathrm{D} / 3 \mathrm{D}$ registration context indicate that one iteration can be done in around $240 \mathrm{~ms}$ and a typical 20-iteration registration in 4.8 seconds. This implementation fulfils the overall execution time for the $2 \mathrm{D} / 3 \mathrm{D}$ registration procedure in a real scenario.

\section{Discussion and Conclusions}

The Siddon-Jacobs algorithm is used as the ray-casting method to render pixels to implement a digitally reconstructed radiograph (DRR) for our intensity-based $2 \mathrm{D} / 3 \mathrm{D}$ registration. The evaluation of the DRR module is carried out using a CT-scan of $512 \times 512 \times 442$ voxels and creating $568 \times 568$ pixels DRR images. The render of a pixel is created using the line integral over the ray-path traversing the DICOM volume. The ray-path calculation takes longer the more voxels it passes through. The evaluation is performed using a position and orientation of the ray-path that causes the ray to cross the main diagonal of the DICOM volume. This transformation is implemented using our novel pixel-step method, which transforms four points. One point is the ray-source; the other three are corners of the rendering plane.

As a basis for performance metrics, we use the DRR module implemented in the medical framework and visualization (MeVisLab). The evaluation of the module gave an average DRR image rendering time of 22 seconds.
Our implementation using parallel computing reaches an average execution time of $10 \mathrm{~ms}$, which is $3000+$ times faster than the ground-truth DRR module. With the parallel DRR runtime, one iteration of the $2 \mathrm{D} / 3 \mathrm{D}$ registration takes around $240 \mathrm{~ms}$, a complete registration of 20 iterations in 4.8 seconds. These times are acceptable for the $2 \mathrm{D} / 3 \mathrm{D}$ registration in a real scenario.

\section{Author Statement}

Research funding: Part of this work is funded by the German Federal Ministry of Education and Research (KMU-innovativ: contract number 13GW0175B). Conflict of interest: Authors state no conflict of interest. Informed consent: Informed consent has been obtained from all individuals included in this study. Ethical approval: The research related to human use complies with all the relevant national regulations, institutional policies and was performed in accordance with the tenets of the Helsinki Declaration, and has been approved by the authors' institutional review board or equivalent committee.

\section{References}

[1] Dorgham O, Fisher M, Laycock S. Accelerated generation of digitally reconstructed radiographs using parallel processing; 2009.

[2] Russakoff D, Rohlfing T, Mori K, Rueckert D, Ho A, Adler J, Maurer $C$. Fast generation of digitally reconstructed radiographs using attenuation fields with application to 2D3D image registration. IEEE Transactions on Medical Imaging;2005;24;11;1441-1454.

[3] Siddon R, "Fast calculation of the exact radiological path for a three-dimensional CT array," Medical Physics, vol. 12, no. 2, pp. 252-255, 1985.

[4] Jacobs F, Sundermann F, De Sutter B, Christiaens M, Lemahieu I. A fast algorithm to calculate the exact radiological path through a pixel or voxel space. Journal of Computing and Information Technology;1998;6;89-94.

[5] Cook S. CUDA Programming: A Developer's Guide to Parallel Computing with GPUs. San Francisco: Morgan Kaufmann Publishers Inc. 2012.

[6] Nvidia. CUDA C ++ Programming Guide. Nvidia 2020. 